\title{
CORRIGENDUM
}

Hidenori Sassa - Takeshi Nishio - Yasuo Kowyama

Hisashi Hirano - Takato Koba - Hiroshi Ikehashi

\section{Self-incompatibility (S) alleles of the Rosaceae encode members of a distinct class of the $T_{2} / S$ ribonuclease superfamily}

in Mol Gen Genet (1996) 250:547-557

Unfortunately, the nucleotide sequence of "primer 7" in the subsection "PCR cloning of genomic fragments of S-RNases" was given as $5^{\prime}$ ACGGGGATGATATATRTGGTTA-3'. It should be $5^{\prime}$ TGGTTAYKATGGTATTTTCATTAAT-3'.

Communicated by H. Saedler

H. Sassa (ब) $\cdot$ T. Koba $\cdot$ H. Ikehashi ${ }^{1}$

Faculty of Horticulture, Chiba University, Matsudo,

Chiba 271, Japan

\section{T. Nishio}

Institute of Radiation Breeding, NLAR, MAFF, Ohmiya-machi,

Naka-gun, Ibaraki 319-22, Japan

Y. Kowyama

Faculty of Bioresources, Mie University, Tsu, Mie 514, Japan

H. Hirano ${ }^{2}$

Department of Molecular Biology, National Institute of

Agrobiological Resources, Kannondai, Tsukuba, Ibaraki 305, Japan

Present addresses:

1 Faculty of Agriculture, Kyoto University, Kita-shirakawa,

Sakyo-ku, Kyoto 606-01, Japan

${ }^{2}$ Kihara Institute for Biological Research, Yokohama City

University, Maioka, Totsuka-ku, Yokohama 244, Japan 